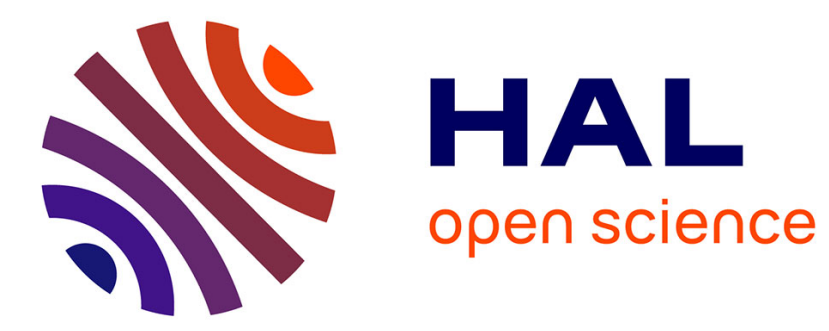

\title{
Les relégués au pénitencier de Saint-Jean-du-Maroni durant le Second Conflit mondial (1939-1943)
}

\author{
Jean-Lucien Sanchez
}

\section{To cite this version:}

Jean-Lucien Sanchez. Les relégués au pénitencier de Saint-Jean-du-Maroni durant le Second Conflit mondial (1939-1943). Sylvie Humbert, Yerri Urban. Histoire de la justice, Justice en Guyane. A l'ombre du droit, La Documentation française, p. 155-170, 2016. halshs-01409114

\section{HAL Id: halshs-01409114 https://shs.hal.science/halshs-01409114}

Submitted on 12 Jan 2018

HAL is a multi-disciplinary open access archive for the deposit and dissemination of scientific research documents, whether they are published or not. The documents may come from teaching and research institutions in France or abroad, or from public or private research centers.
L'archive ouverte pluridisciplinaire HAL, est destinée au dépôt et à la diffusion de documents scientifiques de niveau recherche, publiés ou non, émanant des établissements d'enseignement et de recherche français ou étrangers, des laboratoires publics ou privés. 


\section{Les relégués au pénitencier de Saint-Jean-du-Maroni durant le Second Conflit mondial}

(1939-1943)

Les relégués étaient internés en Guyane française sur le territoire pénitentiaire du Maroni, au pénitencier de Saint-Jean-du-Maroni et dans ses camps annexes. Au début de leur installation, leur taux de mortalité est particulièrement élevé : de 1888 à 1905, il est en moyenne de $12 \%$. Cette situation s'explique essentiellement par leurs conditions d'accueil particulièrement précaires à leur arrivée dans la colonie. L'administration pénitentiaire coloniale n'a pas été en mesure de bâtir les infrastructures destinées à les recevoir et ils doivent construire dans l'urgence des bâtiments pour les héberger eux et leur personnel d'encadrement. Il en résulte des conditions insuffisantes d'hygiène et des épidémies de fièvre jaune, de paludisme et de dysenterie éclatent régulièrement. Ces maladies constituent les principaux facteurs à l'origine de la mortalité élevée des relégués. Néanmoins, à force d'aménagements et d'assainissements, la relégation devient peu à peu salubre et le taux de mortalité annuel oscille, à partir des années 1930, autour de $3 \%$.

Pourtant, à partir de 1941, le taux de mortalité des relégués repart à la hausse pour atteindre un niveau jamais égalé. De 1941 à 1943, il atteint une moyenne de plus de $32 \%$ de l'effectif total. En 1942, c'est près $48 \%$ de l'effectif des relégués qui meurt dans l'année. Cette brusque hausse du taux de mortalité ne s'explique par aucune épidémie particulière. En effet, si l'on compare le taux de mortalité des transportés internés au pénitencier de Saint-Laurent-du-Maroni et dans ses camps annexes, distants de celui de Saint-Jean de seulement $16 \mathrm{~km}$, il est pour la même période de $4 \%$.

Ainsi, qu'est-ce qui explique que sur une aussi courte période les relégués ait été les victimes d'une telle hécatombe ? Comment cette situation a-t-elle pu être possible et qu'est-ce qui l'a générée?

\section{Un régime disciplinaire considérablement durci}

Le régime disciplinaire en vigueur à la relégation avant 1939 est particulièrement drastique. Suite à un mouvement de grève entrepris par les relégués en $1931{ }^{1}{ }^{1}$ le commandant supérieur de la relégation Limouze, spécialement nommé pour en venir à bout, y fait régner une «discipline de fer ${ }^{2}{ }^{2}$ Bien que le décret du 18 septembre 1925 relatif au régime disciplinaire des établissements de travaux forcés aux colonies ait aboli la peine de cachot, les relégués subissent toujours cette peine : la moitié au moins des cellules des bâtiments disciplinaires du pénitencier s'y apparente. Les punitions sont en outre administrées dans des proportions totalement anormales. En 1936, le nombre de journées de cellule subies par les relégués est de 39733 et celui des nuits de prison est

\footnotetext{
${ }^{1}$ Cf. Jean-Lucien Sanchez, A perpétuité. Relégués au bagne de Guyane, Paris, Vendémiaire, 2013, p. 160 et suiv.

2 Le procureur de la République, Rapport sur l'inspection des pénitenciers de la transportation et de la relégation au Maroni, 21 juin 1938, Archives départementales de Guyane (désormais ADG) IX 71.
} 
de 4 644. ${ }^{3}$ Pour le seul mois de février 1938, la commission disciplinaire prononce 13 ans et 4 mois de cellule sans sursis, 56 mois de quartier disciplinaire et 136 jours de privation de promenade. Ces punitions sont de plus subies dans des locaux disciplinaires extrêmement vétustes «qui sont de véritables cachots, obscurs, sans air, extrêmement humides. ${ }^{4} \mathrm{~A}$ cette date, près de $20 \%$ de l'effectif des relégués est interné dans la prison et les locaux disciplinaires du pénitencier qui comprennent 86 cellules et 9 blockhaus.

\section{Une représentation négative des relégués}

Par rapport aux transportés, condamnés en vertu de la loi du 30 mai 1854 sur l'exécution de la peine des travaux forcés, dite loi sur la transportation, les relégués représentent une population bien moins recommandable. Comme le note en 1938 dans un rapport l'inspecteur des colonies Bourgeois-Gavardin : «On a bien souvent fait la comparaison du transporté et du relégué. Elle est évidemment en faveur du premier. Paresse, habitudes invétérées de vol et de vagabondage, dépravation sexuelle extrêmement répandue, tous ces vices du relégué ont été bien souvent établis. ${ }^{5}$

Cette représentation est également partagée par le directeur de l'administration pénitentiaire :

«Il est difficile de corriger le relégué des tares qu'il étale impudemment parfois. Le plus souvent dégénéré, il est resté longtemps en prison. Sa mentalité est la conséquence logique, inévitable de son passé. Le relégué se présente donc sous différents aspects : paresseux, voleur, menteur, débauché, souteneur, escroc le plus souvent; meurtrier plus rarement; quelque fois sous les deux formes. Les penchants qui l'ont amené sur les bancs de la Correctionnelle demeurent en lui, souvent vivaces - quelquefois latents. [...] Une vigilance est indispensable pour maintenir ces individus sous une discipline, dans un esprit de justice et d'équité. Et c'est par là même que l'on serait en droit d'espérer quelque amendement - qui n'est réalisé le plus souvent, à part quelques exceptions, qu'après un temps de probation relativement long - et sans que l'on puisse affirmer qu'il est réel. ${ }^{6}$

Ainsi que par le procureur de la République de la colonie :

«[...] $40 \%$ des relégués sont des dégénérés, ce qu'il y a de certain, c'est que tous sont des individus sans scrupules, parmi lesquels ceux qui se conduisent le moins mal, sont parfois les plus

3 Le ministre des Colonies au gouverneur, 20 janvier 1939, Archives nationales d'outre-mer (désormais ANOM) H 5347.

4 Rapport fait par M. Bourgeois-Gavardin, Inspecteur de $1^{\text {ère }}$ classe des colonies concernant la vérification de M. le sous-directeur de $1^{\text {ère }}$ classe Sontag, directeur par intérim de l'administration pénitentiaire, à Saint-Laurent-du-Maroni, à l'époque du 10 avril 1938, et explications fournies par ce haut fonctionnaire sur les résultats de sa vérification, ANOM H 1877.

5 L'inspecteur de $1^{\text {ère }}$ classe des colonies Bourgeois-Gavardin au ministre des Colonies, 30 juin 1938, ANOM H 1877. 6 Le directeur de l'administration pénitentiaire par intérim Sontag cité dans le rapport fait par M. Bourgeois-Gavardin, Inspecteur de $1^{\text {ère }}$ classe des colonies concernant la vérification de M. le sous-directeur de $1^{\text {ère }}$ classe Sontag, directeur par intérim de l'administration pénitentiaire, à Saint-Laurent-du-Maroni, à l'époque du 10 avril 1938, et explications fournies par ce haut fonctionnaire sur les résultats de sa vérification, ANOM H 1877. 
dangereux qui ont encore assez de force de caractère pour dominer leurs mauvais instincts durant quelque temps afin d'obtenir un relèvement qui leur permettra de recommencer leurs exploits. Seul le costume des transportés et des relégués est le même, tout le reste diffère. ${ }^{7}$

\section{L'exode vers la France Libre}

Les conditions de détention des relégués, marquées par une discipline inflexible et une représentation négative, vont connaître une brusque inflexion à partir de 1941. A la suite de l'armistice du 22 juin 1940, la colonie sous la direction du gouverneur Robert Chot puis sous celle de René Veber reste fidèle au régime de Vichy. Le Conseil général est suspendu et est remplacé par une commission administrative. Aussitôt, une dissidence gaulliste s'organise à l'initiative du capitaine Claude Chandon qui se range à l'appel du général de Gaulle. ${ }^{8}$ Dans la nuit du 5 au 6 février 1941, un mouvement d'évasion de la population pénale se produit sur les pénitenciers du Maroni. Cet «exode » comme le surnomme l'administration pénitentiaire a pour origine un appel lancé depuis la rive hollandaise par Chandon. Celui-ci est un agriculteur installé en Guyane en 1927. ${ }^{9}$ Engagé volontaire en 1939 et démobilisé en août 1940, il rallie la France Libre et quitte la Guyane afin de poursuivre la lutte. Assisté d'un médecin militaire et d'un officier britanniques, il organise depuis la ville d'Albina le recrutement de volontaires. Les recrues sont ensuite dirigées vers le district de Mongo (Guyane anglaise) via Georgetown en attendant leur transfert en Afrique. Leur départ est facilité depuis le village chinois de Saint-Laurent par un agent de liaison de Chandon, le libéré Orliac. L'attitude des autorités hollandaises est néanmoins ambigüe : en présence de Chandon, elles participent activement au recrutement. Mais ce dernier absent, elles renvoient les évadés. De même, les individus reconnus inaptes au service par Chandon sont immédiatement reconduits en Guyane française. Néanmoins, à partir d'octobre 1941, un rapport de l'administration pénitentiaire signale, sur la foi d'un indicateur, que le commissaire de police d'Albina aurait assuré que la reine de Hollande en personne aurait donné l'ordre de ne plus remettre d'évadés aux autorités. De nouveaux convois de forçats à destination des forces alliées seraient donc encore en préparation à cette date et un florin serait payé à chaque Amérindien ou Noir Marron pour le transport d'un évadé. Ceux reconnus aptes seraient toujours dirigés sur Mongo mais ceux reconnus inaptes seraient incarcérés et ne seraient désormais plus remis aux autorités françaises. Le directeur des services pénitentiaires coloniaux écrit alors au commissaire du district de Mongo pour le dissuader de recevoir les relégués :

7 Le procureur de la République, Rapport sur l'inspection des pénitenciers de la transportation et de la relégation au Maroni, 21 juin 1938, ADG IX 71.

8 Rodolphe Alexandre, De Pétain à de Gaulle. La Guyane sous Vichy. 1940-1943, Editions Anne C., Région Guyane, Cayenne, 2003, p. 51.

9 Rodolphe Alexandre, «Du monnervillisme à la résistance de Vichy (1932-1944) à Saint-Laurent-du-Maroni », Actes du colloque Cinquantenaire de la création de Saint-Laurent-du-Maroni, 1949-1999, Cayenne, Région Guyane, Paris, Servédit, 2000, p. 191-206, ici p. 201. 
«[...] Ces relégués, qui pour la plupart sont de dangereux malfaiteurs et récidivistes du vol ne peuvent guère, à mon avis, en raison de leur paresse vous rendre de grands services même comme manœuvres. Le but ardemment recherché par cette catégorie de condamnés, réfractaires à tout travail, est de pouvoir se livrer à leurs vices invétérés le vol et la paresse qui peuvent même les conduire aux crimes car ils ne sont que trop souvent coutumiers du fait. Dans votre intérêt, en vue de protéger vos ressortissants et, pour permettre à la justice d'exercer plus rapidement son droit de répression je vous serais reconnaissant désormais de vouloir bien, comme par le passé, envisager la possibilité de faire reconduire sur le Territoire Français ces individus dangereux véritable rebut de la Société. » ${ }^{10}$

Mais cette information n'est pas confirmée par les services pénitentiaires coloniaux qui pensent plutôt avoir affaire à un nouvel acte de propagande destiné à encourager les évasions de forçats pour rejoindre les forces alliées. ${ }^{11}$ Le soulagement n'intervient qu'en avril 1941, lorsque le chef des services pénitentiaires coloniaux rencontre le commissaire d'Albina qui lui assure que Chandon est définitivement parti accompagné par ses recrues se battre en Afrique. ${ }^{12}$

Le gouverneur de la Guyane française ne saisit pas tout de suite la portée de ces évasions. Le recrutement de ces hommes sert les intérêts des puissances en guerre contre les puissances de l'Axe et sont de ce fait considérées comme des ennemis par le gouverneur fidèle au régime de Vichy. Mais ces nouvelles recrues vont-elles partir se battre en Afrique ou bien vont-elles se retourner contre la Guyane et l'envahir ? Le 8 février 1941, des avions de l'armée américaine patrouillant audessus de Saint-Laurent sont effectivement signalés et le gouverneur redoute une attaque. ${ }^{13}$ En réaction, il décide de doubler les effectifs de surveillance sur les points les plus importants du fleuve. Les sorties de corvées des punis d'emprisonnement et des réclusionnaires sont supprimées et de nouveaux postes de gardes sont installés. Des patrouilles sillonnent jour et nuit le fleuve et des battues d'évadés sont organisées aux abords des pénitenciers de Saint-Jean et de Saint-Laurent. Tous les canots sont interdits de navigation et la pêche est prohibée. Enfin, 160 soldats en provenance de Martinique sont stationnés à Saint-Laurent et la frontière avec la Guyane hollandaise est fermée.

Le 11 février, le chef des services pénitentiaires coloniaux se rend à Saint-Laurent et s'entretient avec des informateurs ainsi qu'avec la plupart des autorités : «Peu à peu, j'ai eu l'impression qu'il n'y avait pas actuellement de menaces d'invasion dirigées contre la Guyane Française. ${ }^{14}$

10 Le lieutenant-colonel Camus au commissaire du district de Mongo, 22 octobre 1941, Musée national des prisons.

11 Direction des services pénitentiaires coloniaux au gouverneur, 20 octobre 1941, ANOM H 5114.

12 Le chef des services pénitentiaires coloniaux au gouverneur, 4 avril 1941, ANOM H 5154.

13 Le commissaire de police au chef des services pénitentiaires coloniaux, 13 février 1941, ANOM H 5114.

14 Le chef des services pénitentiaires coloniaux, Rapport au gouverneur, 18 février 1941, ANOM H 5114. 
Le 13 février, le mouvement est totalement maîtrisé. Les relégués jugés les «plus dangereux » ont été internés au quartier disciplinaire tandis que 252 d'entre eux ont été internés et surveillés étroitement dans six cases du pénitencier. ${ }^{15}$ Les mesures sont draconiennes car la propagation de l'appel de Chandon au reste de la colonie est redoutée. Le 17 février, un libéré informe ainsi le commissaire de police de Saint-Laurent qu'un relégué individuel se serait rendu à Cayenne qui compterait depuis près de 900 volontaires (dont 350 bagnards) prêts au départ. ${ }^{16}$

Suite à cet épisode, le chef des services pénitentiaires coloniaux décide de mener une enquête afin de déterminer les origines de la propagation de l'appel de Chandon au sein des pénitenciers. S'entretenant avec Limouze sur l'évasion de 17 relégués de Saint-Jean, ce dernier reçoit des renseignements précis d'informateurs qui lui auraient assuré que le capitaine de l'Armée du Salut, Pierre Hausdorff, serait à l'origine des évasions produites à la relégation. Hausdorff visite tous les quinze jours Saint-Jean et y organise une réunion d'une demi-heure autour de chants et de prières devant un parterre composé de trente à cinquante relégués. ${ }^{17}$ Un surveillant y assiste normalement. Mais lors de celle tenue le 8 février 1941, profitant de l'absence de surveillance et en vue d'un mouvement massif d'évasion prévu pour le 27 février, il aurait tenu les propos suivants : «Chers concitoyens, n'en veuillez pas à la France d'être dans l'état où vous vous trouvez, la loi des hommes condamne même les petits délits et si vous êtes relégués ne lui en gardez pas rancune, le moment est venu de pouvoir vous libérer; faites le sans scrupule car c'est pour défendre la France. $»^{18}$

Manquant de preuves formelles pour le faire arrêter, le chef des services pénitentiaires coloniaux lui interdit l'accès jusqu'à nouvel ordre de tous les pénitenciers du Maroni et le fait surveiller. Plus tard, en février 1942, Hausdorff et sa famille sont expulsés de Guyane. Selon Charles Péan, cette expulsion permettra à l'administration pénitentiaire de se passer désormais d'un témoin gênant à la relégation : «Une fois l'officier de l'Armée du Salut exécuté, libérée de tout témoin, l'Administration pénitentiaire, saisie d'une véritable folie, déclenche à Saint-Jean un régime de terreur, d'une brutalité inouie : privation de nourriture, travail à coups de trique, de nerf de bœuf. Qu'ils marchent ou qu'ils crèvent ! En quelques mois, la mortalité qui, -sauf à l'arrivée des convoisoscille de trois à cinq pour cent, passe à quarante-six pour cent $! »^{19}$

Par la suite, en juillet 1941, le gouverneur engage le chef des services pénitentiaires coloniaux à se montrer très scrupuleux vis-à-vis des «missions confessionnelles étrangères »

15 Le commandant supérieur du pénitencier de Saint-Laurent au chef des services pénitentiaires coloniaux, 16 février 1941, ANOM H 5154.

16 Le commissaire de police au chef des services pénitentiaires coloniaux, 17 février 1941, ANOM H 5154.

17 Charles Péan, Conquêtes en terre de bagne, Paris, Altis, 1948, p. 315.

18 Le commandant supérieur du pénitencier de Saint-Laurent au chef des services pénitentiaires coloniaux, 13 février 1941, ANOM H 5154.

19 Charles Péan, Conquêtes en terre de bagne, op. cit. 17, p. 317. 
présentes dans la colonie. D'après lui, le département du ministère de l'information à Londres les utiliserait afin de diffuser des tracts favorables à la France Libre, à l'Empire Français Libre et aux gouvernements des puissances européennes occupées en exil à Londres. ${ }^{20}$ En réaction, tous les membres de l'Armée du Salut se voient interdire l'entrée des pénitenciers. ${ }^{21}$ Pourtant, après enquête, l'accusation portée contre Hausdorff semble peu crédible. Dans la soirée du 8 février, l'officier a effectivement tenu une conversation avec des relégués à Saint-Jean. Parmi eux, six acceptent de témoigner: un seul affirme que le capitaine n'aurait pas ouvertement encouragé les relégués à s'évader mais l'aurait néanmoins laissé sous-entendre. Tous les autres affirment que le capitaine aurait tenu des propos d'ordre essentiellement moral et qu'il aurait même enjoint à l'un d'entre eux de prendre son mal en patience plutôt que de tenter une évasion. ${ }^{22}$

En parallèle, un mouvement d'insubordination est projeté par des relégués punis afin de permettre à certains d'entre eux de s'évader pour rejoindre la France Libre. Mais Limouze est alerté par un informateur de cette tentative et prend des dispositions pour l'empêcher. Le 14 février, vers midi, des relégués du quartier disciplinaire se plaignent de leur soupe et débutent une mutinerie. Aussitôt, six surveillants militaires escortés de porte-clefs ${ }^{23}$ armés viennent en renfort du personnel de surveillance du quartier disciplinaire et le mouvement est stoppé en dix minutes. A la suite de quoi, le chef des services pénitentiaires coloniaux décide d'interner provisoirement 225 relégués au pénitencier des Îles du Salut et Limouze informe les relégués que tout individu qui prend ou conserve un service auprès d'une armée étrangère est désormais passible de la peine de mort au titre de trahison. ${ }^{24}$

En tout, 70 relégués collectifs se sont évadés. Sur ce nombre, 56 ont été réintégrés. ${ }^{25}$ A ce chiffre s'ajoute 11 relégués individuels et 6 relevés de la relégation tous partis entre le 5 et le 10 février. Il semble que Chandon ne soit parvenu à organiser qu'un seul convoi de condamnés en direction de l'Afrique. ${ }^{26}$ Après son départ, d'autres évadés tenteront encore de rejoindre les forces de la France Libre mais la plupart finiront dans des camps de concentration, à Cuba ou à Trinidad notamment, et ce jusqu'à la fin des hostilités.

20 Télégramme du gouverneur au chef des services pénitentiaires coloniaux, $1^{\text {er }}$ juillet 1941, ANOM H 2081.

21 Le chef des services pénitentiaires coloniaux au gouverneur, 16 août 1941, ANOM H 2081.

22 Procès-verbaux d'informations des relégués collectifs Oubot, Dupont, Peldarre, Alix, Gomberte et Merck dressés par le surveillant chef Marcel Dalissier et transmis au chef des services pénitentiaires coloniaux le 26 mars 1941, ANOM H 5154 .

23 C'est-à-dire des relégués auxiliaires de l'administration pénitentiaire.

${ }^{24}$ Par la loi du 27 juillet 1940, les dispositions du décret du 29 juillet 1939 portant condamnation à la peine de mort pour trahison sont étendues à tout Français qui sans l'autorisation du gouvernement français prend ou conserve un service dans une armée étrangère ou fait l'enrôlement pour une puissance étrangère, le chef des services coloniaux, 22 février 1941, Archives municipales de Saint-Laurent-du-Maroni.

25 Le chef des services pénitentiaires coloniaux au gouverneur, 19 février 1941, ANOM H 5114.

${ }^{26}$ Le transporté Charles Hut affirme que seuls 80 transportés parvinrent à rallier la France Libre et à rejoindre Brazzaville, $c f$. René Delpêche, Parmi les fauves et les requins, ou la confession de M. Charles Hut, ancien forçat, Paris, J. d'Halluin, 1955, p. 168. 


\section{La réaction à « l'exode »}

Après l'épisode de « l'exode », l'inspecteur des colonies Devouton est spécialement diligenté en Guyane afin d'y faire échec à la «propagande anti-française sur le territoire pénitentiaire du Maroni ». ${ }^{27}$ L'inspecteur a surtout à cœur d'empêcher l'entrée des journaux et des tracts de la France Libre édités depuis la Guyane hollandaise. L'organe principal de la France Libre est le journal France. Un Britannique, propriétaire d'un magasin à Albina, se charge de recevoir les journaux et les tracts et les diffuse ensuite à Saint-Laurent. Il profite du va et vient des sujets hollandais qui se rendent fréquemment à Saint-Laurent pour y procéder à des achats. Alors que les déplacements en direction de la Guyane hollandaise sont strictement encadrés pour les Guyanais, les sujets Hollandais ne sont astreints à aucune restriction de déplacement, car la Guyane française est en grande partie dépendante du ravitaillement opéré par les navires hollandais durant la guerre. Dorénavant, tout sujet hollandais doit débarquer obligatoirement sur un point fixe de la colonie contrôlé par des douaniers chargés d'empêcher l'entrée de tracts et d'armes. ${ }^{28}$

De même, le contrôle postal devient scrupuleux et tous les courriers et télégrammes à destination ou au départ de Saint-Laurent sont systématiquement ouverts : ceux en langue étrangère, en particulier en anglais, sont traduits et lus par les autorités françaises à l'affût de toute propagande « antinationale ». Une commission de contrôle postal et télégraphique censure un grand nombre de courriers expédiés ou reçus par des particuliers, des condamnés ou des membres des services pénitentiaires coloniaux. Elle signale au gouverneur ceux qu'elle juge tendancieux et censure ceux contenant toute allusion à la situation de pénurie de ravitaillement de la colonie, à la dissidence gaulliste au sein du corps des surveillants militaires ${ }^{29}$ et les propos hostiles au gouvernement de Vichy. ${ }^{30}$

En parallèle, certaines mesures prises au Maroni renseignent sur l'état d'esprit des autorités vis-à-vis des condamnés. L'ordre est par exemple donné d'arrêter et de mettre en résidence surveillé tous les ressortissants russes. ${ }^{31}$ Un recensement indique que sur les dix ressortissants russes résidant en Guyane, tous sont d'origine pénale. Parmi eux, deux transportés en cours de peine ont déjà été condamnés à mort par le tribunal maritime spécial pour évasion et tentative d'enrôlement dans une armée étrangère. Quatre autres sont des transportés libérés en état d'arrestation et quatre derniers

27 Le chef des services pénitentiaires coloniaux au gouverneur, 2 août 1941, ANOM H 2081.

28 L'inspecteur de $1^{\text {ère }}$ classe des colonies Devouton au sous-secrétaire d'Etat aux Colonies, 21 juillet 1941, ANOM H 2081.

29 Le transporté Emile Jusseau affirme que des surveillants ralliés à la France Libre auraient aidé des forçats à traverser le Maroni pour rejoindre Chandon et auraient organisé des mutineries au pénitencier de la transportation avec la complicité de transportés, $c f$. Emile Jusseau, Les cloches de la camarde. 24 de survie au bagne de Cayenne, Nyons, A. Chantemerle, 1974, p. 267-268.

30 Rapport de l'administrateur des colonies chargé de l'expédition des affaires courantes des services pénitentiaires coloniaux au gouverneur, 10 décembre 1940, ANOM H 5154.

31 Le chef des services pénitentiaires coloniaux au gouverneur, 8 juillet 1941, ANOM H 5114. 
sont des relégués collectifs. Ceux-ci sont immédiatement transférés au pénitencier des Îles du Salut pour y être soumis à une surveillance spéciale.

De même, en juillet 1941, l'inspecteur des colonies Devouton propose d'interner dans un camp de concentration les relégués relevés de leur peine et les transportés libérés indigents. La guerre ayant stoppé les convois de rapatriement, ils sont nombreux à mener une existence de misère dans les rues de Cayenne ou de Saint-Laurent. ${ }^{32}$ L'inspecteur propose donc d'interner dans un camp de concentration les individus reconnus valides, libérés depuis plus de trois mois et ne justifiant pas de moyens d'existence normaux ${ }^{33}$. En plus de vouloir interner les libérés dans un camp de concentration, le maire de Saint-Laurent fait procéder au recensement de tous les Juifs présents sur le territoire pénitentiaire. L'article 11 de loi du 2 juin 1941 portant statut des Juifs étend effectivement ses dispositions aux colonies. Dès qu'il reçoit le Journal Officiel de la Guyane Française en date du 28 juin 1941, le maire fait publier au son du tambour et afficher en ville le texte de la loi. Cinq «déclarations de race et de religion juives ${ }^{34}$ sont souscrites : trois par des relégués individuels et deux par des transportés libérés. Mais la loi s'applique à tous les Juifs, fonctionnaires coloniaux inclus. Sur ordre du gouverneur, 46 fonctionnaires, agents et employés des services pénitentiaires coloniaux et employés communaux de Saint-Laurent souscrivent une déclaration selon laquelle ils ne sont pas de confession juive.

\section{Un régime considérablement durci}

L'entrée en guerre et les pénuries qui en découlent frappent durement les relégués mais également leur personnel d'encadrement. De 65 en 1939, le nombre de surveillants n'est plus que de 54 en décembre 1940. Le régime de leur solde remonte à 1931 et celui de leurs indemnités à 1928 : un surveillant de $1^{\text {ère }}$ classe gagne 1000 francs mensuels et un surveillant de $3^{\text {ème }}$ classe gagne 847 francs mensuels. ${ }^{35}$ Ces faibles rémunérations et les privations dues aux hostilités ne leur permettent pas de faire vivre correctement leur famille. Ils ne parviennent pas par exemple à acheter des chaussures à leurs enfants qui sont contraints d'aller pieds nus dans les rues. Certains se couvrent de dettes ou bien chassent en dehors de leurs heures de travail, non pour leur plaisir, mais afin de nourrir leur famille. La plupart sont de plus très inquiets par la situation de leurs proches restés en métropole durant le Second Conflit mondial. La discipline se relâche et le commandant doit faire montre d'une grande sévérité à leur encontre : «La discipline a dû être reprise avec plus de sévérité,

\footnotetext{
${ }^{32}$ Cf. Danielle Donet-Vincent, De soleil et de silences. Histoire des bagnes de Guyane, Paris, La Bibliothèque de l'Histoire, 2003, p. 350.

33 Note au gouverneur, 10 juillet 1943, ANOM H 5114.

34 Le chef des services pénitentiaires coloniaux au gouverneur, 26 août 1941, ANOM H 5114.

35 Rapport fait par M. Lebegue, inspecteur de $1^{\text {ère }}$ classe des colonies concernant la vérification de M. le docteur Sainz, médecin lieutenant-colonel des troupes coloniales hors cadres, directeur des services pénitentiaires coloniaux à SaintLaurent-du-Maroni, à la date du $1^{\text {er }}$ mai 1946 et explications fournies par cet officier supérieur sur le résultat de sa vérification, ANOM H 1877.
} 
certains agents affectés par le manque de nouvelles de la métropole s'étaient laissés aller à un relâchement dans leur service. $»^{36}$

La conséquence de cette sévérité se traduit par le nombre assez élevés de punitions prononcées contre les surveillants en 1940 : 118 jours d'arrêts de rigueur, 147 jours d'arrêts simples et 2 mois de suspension de solde. Mais si le régime est dur pour le personnel des services pénitentiaires coloniaux, il l'est bien plus encore pour les relégués. La discipline est toujours aussi drastique à Saint-Jean et la commission disciplinaire inflige en 1942832 mois de quartier cellulaire et 44356 jours de cellule. Cette rigueur disciplinaire s'accompagne en outre de décisions qui durcissent considérablement le régime en vigueur à la relégation. En mars 1941, le gouverneur décide de rétablir la peine de pain sec abolie pourtant en 1925. Dorénavant, les relégués punis de cellule et non malades sont mis au pain sec un jour sur trois. Cette mesure va avoir des conséquences dramatiques sur l'état général de santé des relégués. Car le régime cellulaire devient le régime commun de la majorité d'entre eux à partir de 1940: en 1941, près de la moitié de l'effectif est emprisonné ! Cette réaction est notamment motivée par la volonté du gouverneur de contrevenir à «l'exode» des relégués à la suite de l'appel de Chandon : «Enrayer d'une façon radicale l'indiscipline latente qui règne actuellement au bagne et qui a été provoquée par les évènements de ces jours derniers dus à l'activité néfaste de l'ex-capitaine CHANDON. » ${ }^{37}$

Dans la même optique, la loi du 4 mars 1942 modifie l'article 14 de la loi du 27 mai 1885. Dorénavant, tout relégué coupable d'évasion ou de tentative d'évasion ou qui sans autorisation quitte la colonie est traduit devant le tribunal correctionnel et est automatiquement condamné à une peine de six mois à un an de prison. En cas de première récidive, la peine est automatiquement portée de un à deux ans de prison et, pour toutes les suivantes, de deux à cinq ans. ${ }^{38}$

Les conditions de travail des relégués sont également durcies à partir de 1942. Le décret du 6 mai 1938 encourageait le travail à la tâche à la relégation : il permettait à un relégué de bénéficier de tout son temps de libre une fois cette dernière exécutée. Lorsqu'il n'était pas employé à la tâche, la journée de travail du relégué était limitée à 6 heures quotidiennes. L'arrêté du $1^{\text {er }}$ septembre 1942 prévoit à l'inverse que le travail à la tâche ne soit autorisé pour les relégués que sur les chantiers forestiers. ${ }^{39}$ Pour tous les autres, la durée de travail quotidienne passe à huit heures. En parallèle, face à l'augmentation du prix des denrées, le département des Colonies ne parvient plus à subvenir aux besoins indispensables à l'alimentation des condamnés. En mars 1940, l'administrateur des

36 Relégation, Rapport annuel, 1940, ANOM H 5147.

37 Le gouverneur au secrétaire d'Etat aux Colonies, 21 mars 1941, ANOM H 2070.

38 Loi n³44 du 4 mars 1942 portant modification de l'article 14 de la loi du 27 mai 1885 sur les récidivistes, ANOM H 1942.

39 Arrêté du $1^{\text {er }}$ septembre 1942 portant modification à l'article 1er de l'arrêté local n420 du 6 mai 1938 organisant le travail des relégués collectifs, ANOM H 1946. 
colonies chargé de l'expédition des affaires courantes de l'administration pénitentiaire, Dimbour, ordonne de faire montre de la «plus grande économie ${ }^{40}$ vis-à-vis des vivres et de toutes matières, produits et denrées reçus de métropole au titre des chapitres hôpitaux, matériel, habillement et couchage. En conséquence de quoi, toutes les rations alimentaires sont diminuées et les suppléments de ration réduits des deux tiers et considérés comme « une récompense exceptionnelle pour un travail effectivement exceptionnel et soutenu. » ${ }^{41}$

Les conséquences des hostilités se font également sentir au niveau de l'habillement des relégués. Ordre est donné de faire durer le plus longtemps possible leurs effets afin de réduire leur délivrance au strict minimum. Par exemple, les punis sont pourvus de vêtements confectionnés exclusivement avec des sacs de farine en toile de coton. La taille des couvertures allouées est même réduite et la quantité mensuelle de savon passe de 400 à 200 grammes. $^{42}$

\section{Le « syndrome cachectique » des relégués}

Ce régime est d'une telle rigueur qu'il entraîne un taux de mortalité très important parmi les relégués punis. En janvier 1943, le chef du service de santé de la colonie, le médecin chef Meydieu, décrit dans un rapport le «syndrome cachectique » dont souffrent nombre de relégués. D'après le médecin, ce syndrome est généré par la durée et la répétition du régime cellulaire et par l'insuffisance du régime alimentaire :

«- Pâleur extrême des téguments parfois, lividité;

- Amaigrissement considérable avec œdèmes et bouffissure des membres inférieurs ou de la face (plus ou moins accusés);

- Anémie profonde (numérotation globulaire descendant parfois jusqu'à 1800000 globules rouges);

- Diarrhée profuse (fréquente, mais non constante). ${ }^{43}$

De son côté, le médecin de Saint-Jean ne peut quasiment rien faire pour enrayer cette situation, car les médicaments manquent. D’après lui, un relégué meurt en moyenne chaque jour au pénitencier. Un tiers environ du total des relégués sont dans des conditions physiques «absolument lamentables $»^{44}$ et sont considérés en état de misère physiologique. Notamment ceux maintenus en prison car sur environ 760 relégués présents à Saint-Jean au 6 février 1942, 340 sont enfermés en

40 Circulaire $n^{\circ} 31$ du chef des services pénitentiaires coloniaux à titre provisoire au sujet de l'alimentation du bagne et économie sur les vivres, 7 mars 1940, ANOM H 2075.

41 Circulaire $n^{\circ} 70 \mathrm{du}$ chef des services pénitentiaires coloniaux à titre provisoire au sujet des réductions apportées aux rations de vivres des transportés, relégués collectifs et déportés. Accroissement de la production de la main-d'œuvre pénale, 20 juin 1940, CAOM H 2075.

42 Circulaire $\mathrm{n}^{\circ} 81 \mathrm{du}$ chef des services pénitentiaires coloniaux à titre provisoire au sujet des mesures nouvelles concernant l'habillement et le couchage des relégués, 26 juillet 1941, ANOM H 2075.

43 Le médecin chef de $2^{\text {ème }}$ classe Meydieu au gouverneur, 29 janvier 1943, ANOM H 5125.

44 Le médecin capitaine des troupes coloniales chargé du service médical du pénitencier de la relégation au lieutenantcolonel directeur par intérim des services pénitentiaires coloniaux, 4 février 1942, ANOM H 5125. 
prison. En décembre 1942, Meydieu prend des mesures de première urgence : il décide de séparer les relégués en trois groupes (A, B et C) selon les aptitudes physiques de chacun. Seul le groupe A est reconnu apte à tous les travaux et doit être séparé des deux autres groupes. Ces mesures sont conduites par un médecin accompagné d'un microbiologiste de l'Institut Pasteur qui opère des prélèvements sur les relégués. Fort des conclusions de l'Institut Pasteur, Meydieu préconise de veiller à ce que la ration des relégués soit intégralement délivrée, de distribuer de la viande fraîche, d'accorder une douche quotidienne, de distribuer des vêtements et des chaussures convenables, de délivrer du papier hygiénique, d'interdire d'employer l'eau du puits pour la cuisine, de réduire la durée de travail des relégués du groupe A et de faire distribuer du lait de chaux ferrugineux.

Mais le gouverneur ne partage pas les vues du médecin :

« J'ajoute que ce n'est vraisemblablement pas pendant les heures de travail pour l'Administration où les relégués sont sous la surveillance du personnel mais bien plus pendant les heures de travail pour la «camelote » et la «débrouille » qui n'a pas d'autre but que de permettre aux relégués de satisfaire leurs vices et leurs instincts anormaux ou de faciliter leurs tentatives particulièrement débilitantes d'évasion que leur santé s'altère. Pour se procurer des papillons, l'osier, le raphia et les bois durs nécessaires à la confection de petits objets destinés à leur fournir des fonds pour des fins inavouables ou criminelles les relégués ne prennent aucun soin des conditions sanitaires des sols marécageux qu'ils doivent traverser, de la qualité de l'eau qu'ils boivent alors, ni aucune mesure d'hygiène et c'est à ce moment surtout qu'ils contractent les affections qui inquiètent. $\gg^{45}$

Rejetant l'idée d'améliorer la ration alimentaire allouée aux relégués au moment où «la population non pénale se voient imposer des restrictions », le gouverneur réclame essentiellement la répression de toute activité des relégués en dehors de leurs heures de travail.

Il ordonne même le retour à Saint-Jean durant l'été 1942 de tous les relégués dispersés dans d'autres pénitenciers, malades inclus. Ils sont effectivement 45 relégués internés au pénitencier de Saint-Laurent, 37 dans des camps annexes (Malgaches et Charvein), 87 aux Îles du Salut et quelques-uns à Cayenne. Il justifie ce regroupement pour des raisons purement économiques. Notamment en ce qui concerne les îles du Salut qui, bien qu'elles constituent le pénitencier le plus salubre de toute la colonie, coûtent trop cher en ravitaillement et en personnel. Le gouverneur connaît pourtant parfaitement le sort des relégués et l'effroyable taux de mortalité qui sévit à SaintJean puisqu'il s'y rend en tournée durant l'été 1942. Mais la piètre représentation qu'il nourrit à leur endroit le dispense d'agir pour les soulager :

«La visite de l'ambulance, d'ailleurs bien tenue, a permis de constater le grand nombre de 
malades hospitalisés dont beaucoup étaient des habitués de l'hospitalisation préférant envenimer les plaies et se sous-alimenter plutôt que de travailler. Si le nombre de décès est important, le médecin a pu constater qu'il était surtout parmi les évadés et ceux qui pratiquant toutes les tares sociales s'exposent à un véritables épuisement de toutes les facultés physiques de l'individu. La moralité du dernier convoi de 1938-39 semble à ce point de vue atteindre le fond de l'abîme. » ${ }^{46}$

Malgré les dénégations du gouverneur, de nombreux relégués continuent de mourir de misère physiologique et de cachexie à Saint-Jean. Ce syndrome de misère physiologique évolue vers une anémie progressive, une cachexie terminale puis la mort. Ce syndrome est à l'origine de la majorité des décès qui endeuille dans des proportions jamais atteintes jusque-là la relégation à partir de 1941. De 1839 relégués en 1939, leur nombre passe effectivement à 683 en 1943 :

\begin{tabular}{|l|l|l|l|}
\hline Année & Effectif moyen annuel des relégués & Décès & Pourcentage \\
\hline 1939 & 1839 & 75 & 4,07 \\
\hline 1940 & 1607 & 95 & 5,91 \\
\hline 1941 & 1424 & 175 & 12,28 \\
\hline 1942 & 1068 & 513 & 48,03 \\
\hline 1943 & 683 & 248 & 36,31 \\
\hline 1944 & 465 & 15 & 3,22 \\
\hline 1945 & 344 & 8 & 2,03 \\
\hline
\end{tabular}

Source : Rapport fait par M. Perreau, professeur des facultés de droit, attaché à la mission d'inspection des colonies, concernant le service de M. le médecin lieutenant-colonel Sainz, directeur des services pénitentiaires coloniaux, à la date du 30 mars 1946 et explications fournies par cet officier supérieur, ANOM H 1877.

Ainsi, le taux de mortalité pour la seule année 1942 est de 48,03 \% de l'effectif total. Cette situation est propre et exclusive à la relégation car, si on compare avec les transportés, leur taux de mortalité atteint pour la même année $2,81 \%$ de l'effectif : 


\begin{tabular}{|l|l|l|l|}
\hline Année & Effectif moyen annuel des transportés & Décès & Pourcentage \\
\hline 1939 & 2737 & 96 & 3,51 \\
\hline 1940 & 2415 & 73 & 3,06 \\
\hline 1941 & 2137 & 68 & 3,18 \\
\hline 1942 & 1904 & 54 & 2,81 \\
\hline 1943 & 1510 & 93 & 6,16 \\
\hline 1944 & 1332 & 43 & 3,29 \\
\hline 1945 & 1168 & 21 & 1,80 \\
\hline
\end{tabular}

Source : Rapport fait par M. Perreau, professeur des facultés de droit, attaché à la mission d'inspection des colonies, concernant le service de M. le médecin lieutenant-colonel Sainz, directeur des services pénitentiaires coloniaux, à la date du 30 mars 1946 et explications fournies par cet officier supérieur, ANOM H 1877.

Cette dissymétrie est la conséquence directe du régime disciplinaire et alimentaire particulièrement éprouvant rencontré par les relégués durant toute la période du Second Conflit mondial :

«L'augmentation du nombre de décès, particulièrement au camp de Saint-Jean, a été due à l'application stricte, purement littérale, de certain règlement pénitentiaire, application faite vraisemblablement sans parti pris de cruauté mais à coup sûr sans le moindre sentiment d'humanité. Les condamnés qui ne réalisaient pas la tâche journalière qui leur était assignés se voyaient soumis à un régime qui, matériellement, les mettait très rapidement en état de déficience et, moralement, les conduisait parfois au suicide. ${ }^{47}$

D'aspect extérieur, les relégués atteints de «syndrome cachectique » présentent tous un état de maigreur extrême et une fatigue très prononcée, comme en témoigne Charles Péan :

«De temps à autre, on peut voir un étrange cortège gagner l'hôpital de Saint-Laurent. Un garçon dirige avec précaution un groupe d'hommes affreusement maigres, entourant un charreton qui transporte des relégués moribonds en provenance de Saint-Jean et débarqués par chaland à StLaurent. Ceux qui peuvent se tenir debout et marcher, car les places dans la charrette sont limitées, donnent l'impression qu'ils vont choir à chaque pas. Le surveillant, craignant de ne pouvoir les conduire jusqu'au bout, s'écrie de temps en temps :

47 Rapport fait par M. Perreau, professeur des facultés de droit, attaché à la mission d'inspection des colonies, concernant le service de M. le médecin lieutenant-colonel Sainz, directeur des services pénitentiaires coloniaux, à la date du 30 mars 1946 et explications fournies par cet officier supérieur, ANOM H 1877. 
- Appuyez-vous les uns contre les autres pour ne pas tomber ! ${ }^{48}$

L'administration pénitentiaire ne s'explique toutefois pas l'origine de ce mal mystérieux. Sans être en être vraiment convaincues, les autorités pensent dans un premier temps que ce syndrome serait provoqué par les privations que s'imposeraient les relégués punis, comme l'échange de leur ration de pain contre du tabac. L'hypothèse de l'empoisonnement général est ensuite sérieusement envisagée : des prélèvements de selle, de sang et de l'eau du puits de la relégation sont analysés et certains relégués, ainsi que tous ceux employés à l'ambulance et aux cuisines, sont l'objet d'une surveillance particulière. Mais rien n'y fait.

Un timide et tardif effort est entrepris au mois de juin 1942. Il fait suite à un premier aménagement du même ordre introduit par le chef des services pénitentiaires coloniaux en février 1942. Après la mort de 64 relégués au mois de mai 1942, le nouveau chef des services pénitentiaires coloniaux, Lassauguette, consent à assouplir à minima les conditions d'incarcération à Saint-Jean. Les punis d'emprisonnement ou de cellule ont droit à deux promenades quotidiennes en dehors du quartier disciplinaire et le médecin doit les examiner chaque semaine afin de déterminer si le régime du pain sec leur est applicable : «A mon avis, il convient d'interpréter les dispositions du décret du 18 septembre 1941 d'une façon très libérale tant que l'état sanitaire général n'aura pas été nettement amélioré. ${ }^{49}$

De son côté, le gouverneur ordonne en juillet 1942 une enquête sur la situation du pénitencier des Îles du Salut afin d'y envisager un repli éventuel de la relégation. Plus tard, en janvier 1943, il envisage le même repli mais cette fois-ci au pénitencier de Saint-Laurent. Non pas que cet agent s'émeuve du sort des relégués, mais vu à la vitesse à laquelle les effectifs diminuent, Saint-Jean risque rapidement de se vider de ses pensionnaires et de coûter plus cher qu'il ne rapporte. Le gouverneur est effectivement soucieux de procéder à toutes les économies utiles. A cette fin, les services pénitentiaires coloniaux procèdent à un recensement de la population des relégués et le gouverneur effectue alors le calcul suivant : en comptant une moyenne mensuelle de 40 morts, le nombre de relégués encore en vie à Saint-Jean risque d'être inférieur à 400 à la fin de l'année 1943 :

«Dans ces conditions, j'estime qu'à cette époque au plus tard, le maintien d'un camp spécial de la relégation totalement incapable d'assurer sa propre vie par lui-même ne se justifiera plus avec les nombreuses sujétions qu'il impose. En effet, outre l'étendue de ce camp, étiré sur plusieurs kilomètres et son éloignement de Saint-Laurent, son maintien imposerait celui de servitudes importantes, moins justifiables que jamais dans les circonstances actuelles; [...]. » ${ }^{50}$

48 Charles Péan, Conquêtes en terre de bagne, op. cit. 17, p. 317.

49 Note du chef de bataillon Lassauguette, chef des services pénitentiaires coloniaux, $1^{\mathrm{er}}$ juin 1942 , ANOM H 5125.

50 Le gouverneur au chef des services pénitentiaires coloniaux, 25 janvier 1943, ANOM H 5125 
Il décide donc d'organiser le repli de la relégation sur Saint-Laurent à la fin de l'année 1943 et ordonne aux services pénitentiaires coloniaux d'organiser la réception d'environ 500 relégués. Ainsi, plutôt que d'accélérer l'évacuation des relégués et de sauver ceux encore en vie, le gouverneur s'accommode de la perte à prévoir de près de 300 d'entre eux. Mais le 16 mars 1943, la Guyane rallie la France Libre. ${ }^{51}$ Un nouveau gouverneur, Jean Rapenne, approuvé par le Comité Français de Libération Nationale, entre en service à Cayenne et remplace René Veber. Rapenne décide de mettre rapidement un terme à la mortalité des relégués : en mars 1943, un premier contingent de 65 hommes est envoyé aux Îles du Salut et des mesures d'urgence sont prises pour leur venir en aide. ${ }^{52}$ A partir de juillet 1943, tous les relégués sont transférés aux Îles du Salut. Seuls ceux hospitalisés sont maintenus à l'hôpital de Saint-Laurent. Enfin, le pénitencier de Saint-Jean est officiellement supprimé en septembre 1943.

Une ordonnance promulguée le 26 avril 1944 décide d'abroger l'article 4 du décret du 16 janvier 1929: le territoire alloué à la relégation n'est dorénavant plus situé au Maroni mais exclusivement sur l'île Royale et sur l'île Saint-Joseph. Au mois d'octobre 1944, ils sont ainsi 194 relégués concentrés sur l'île Royale et 2 installés sur l'île du Diable. ${ }^{53}$ Ils demeurent sous une latitude clémente où la plupart sont classés aux travaux légers. Ce qui entraîne une chute de leur taux de mortalité à 3,22 \% de l'effectif en 1944. L'île Royale reste ainsi en activité jusqu'à la décision, prise dans le courant de l'année 1946, de procéder au regroupement de tous les condamnés en cours de peine à Saint-Laurent. Le $1^{\mathrm{er}}$ mai 1946, le pénitencier des Îles du Salut est à son tour officiellement fermé et les relégués sont transférés au pénitencier de Saint-Laurent. Ils y resteront jusqu'en août 1953, date du départ de l'ultime convoi de rapatriement vers la métropole des derniers bagnards encore en cours de peine.

\section{Conclusion}

Un régime disciplinaire extrêmement sévère couplé à une représentation négative des relégués constituent le quotidien de leur détention avant le déclenchement de la Seconde Guerre mondiale. Mais dans le cadre de l'état d'exception qui règne à la relégation à partir de 1941, ces facteurs connaissent une brusque inflexion et entraînent un taux de mortalité tout à fait unique dans les annales du bagne de Guyane. Les restrictions imposées par la Métropole, l'inquiétude suscitée par la dissidence gaulliste auprès du gouverneur et la situation économique critique rencontrée par le personnel de surveillance à Saint-Jean sont autant de facteurs qui permettent d'expliquer le traitement administré aux relégués durant le Second Conflit mondial. Leur pénitencier n'étant pas

51 Cf. Ulrich Sophie, Le ralliement de la Guyane à la France Libre (16-17 mars 1943), Paris, Editions Louis Soulanges, 1964, p. 105-148.

52 Le commandant du pénitencier des Îles du Salut au chef des services pénitentiaires coloniaux, 13 mars 1941, ANOM H 5154.

53 Pénitencier de la relégation, îles du Salut, mois d'octobre 1944, ANOM H 5150. 
clôturé (à l'inverse de celui des transportés à Saint-Laurent), les autorités ont procédé à leur internement dans des locaux disciplinaires inadaptés et les ont considérés comme des « ennemis de l'intérieur ». Mais outre ces facteurs, la représentation particulièrement négative des relégués qui préexistait avant-guerre s'est radicalisée durant la période d'instabilité ouverte par les hostilités. Le fait qu'ils aient été considérés comme des «tarés » et des «dégénérés » par l'administration pénitentiaire coloniale n'a ainsi fait que renforcer et faciliter l'abandon dans lequel ils ont été précipités. Tout en la retenant d'agir pour les soustraire de la mort certaine à laquelle elle les avait voués.

Jean-Lucien Sanchez 